\title{
Sticky Rouse Time Features the Self-Adhesion of Supramolecular Polymer Networks
}

\section{Journal Article}

\section{Author(s):}

Shen, Zhiqiang; Ye, Huilin; Wang, Qiming; Kröger, Martin (D); Li, Ying

Publication date:

2021-06-08

Permanent link:

https://doi.org/10.3929/ethz-b-000492820

Rights / license:

Creative Commons Attribution-NonCommercial-NoDerivatives 4.0 International

Originally published in:

Macromolecules 54(11), https://doi.org/10.1021/acs.macromol.1c00335

\section{Funding acknowledgement:}

185052 - VISIONNANO - Viscoelastic properties, entanglements, and polymer dynamics in ionic nanocomposites (SNF) 


\title{
Sticky Rouse Time Features the Self-Adhesion of Supramolecular Polymer Networks
}

\author{
Zhiqiang Shen, Huilin Ye, Qiming Wang, Martin Kröger, and Ying Li* \\ Cite This: Macromolecules 2021, 54, 5053-5064 \\ Read Online
}
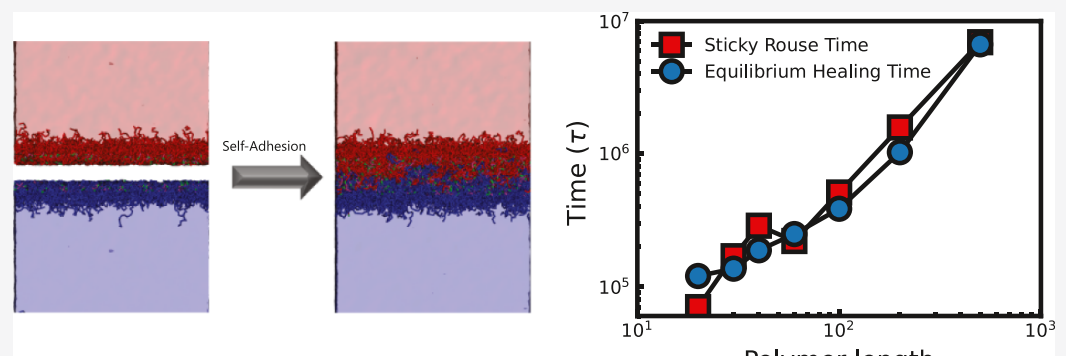

Polymer length

ABSTRACT: Supramolecular polymers are fascinating materials due to their strikingly self-healing capabilities empowered by reversible bonds. However, due to the lack of knowledge about the molecular structure evolution at the fractured interfaces, there is no existing theory to explain and predict the diverse healing times of different supramolecular materials observed in experiments. Here, we systematically study the self-adhesion of both unentangled and entangled supramolecular polymer networks through molecular simulations. We find that the recovery of macroscopic interfacial strength almost linearly depends on the microscopic molecular formations at fractured interfaces of supramolecular polymers, including reversible bonds and entanglements (entangled systems only). More importantly, we place the healing time into the context of intrinsic relaxation timescales of supramolecular polymer networks. It is found that the intrinsic sticky Rouse time features the self-adhesion process of all fractured supramolecular polymers, representing the full recovery of interfacial strength. At this critical timescale, two things happened to guarantee the full recovery of fractured systems: (i) polymer chains have diffused across the fractured interface with a displacement comparable to their sizes; (ii) the crossed stickers and polymer chains have updated their reversible bonds and entanglements (entangled systems only), respectively. The clear molecular description and suggested characteristic self-adhesion time will help the molecular design of supramolecular polymers.

\section{INTRODUCTION}

To mimic the self-repairing capability of human muscles and skins, numerous synthetic self-healable supramolecular polymers have been recently developed. ${ }^{1,2}$ The strikingly selfhealing properties of these polymers arise from reversible bonds ("r-bonds") that cross-link polymer chains as a dynamic network. $^{3-5}$ These r-bonds can autonomously reform after breaking or dissociation, ${ }^{6}$ which can be achieved through dynamic covalent bonds, ${ }^{7}$ hydrogen bonds, ${ }^{1}$ metal-ligand coordination, ${ }^{8}$ hydrophobic interactions, ${ }^{9}$ and ionic interactions. ${ }^{10}$ As a result of these r-bonds, supramolecular polymers can repair fracture or damage at the molecular or microscopic scale and restore their mechanical strength at the macroscopic scale. These polymers have been applied to a wide range of engineering applications, including flexible electronics, ${ }^{11}$ energy storage devices, ${ }^{12}$ biomaterials, ${ }^{13}$ soft robotics, ${ }^{14}$ and lattice structures. ${ }^{15}$

Considering their versatility, it is of paramount importance to study their required healing time and recovered interfacial strength for fractured supramolecular polymers. However, due to different r-bonds and polymer chains used in experiments, the reported characteristic healing times cover a wide range of values from a few seconds to a few days. For instance, Sitti and co-workers ${ }^{16}$ designed self-healing materials by programming biosynthetic proteins with tandem repeat peptides. These synthetic proteins can form a supramolecular network with flexible chains dynamically cross-linked by $\beta$-sheets. Through local heating above $43{ }^{\circ} \mathrm{C}$ near the fracture, their polypeptide materials can self-heal in a second with a mechanical strength of 2-23 MPa. Leibler and co-workers ${ }^{1}$ used fatty dimer acids to fabricate supramolecular material networking by hydrogen bonds between small molecules. The interfacial strength of their materials can recover to $2.5 \mathrm{MPa}$ after a $6 \mathrm{~h}$ healing

Received: February 11, 2021

Revised: $\quad$ May 5, 2021

Published: May 20, 2021

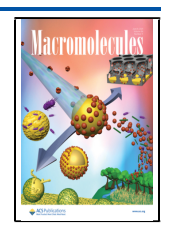



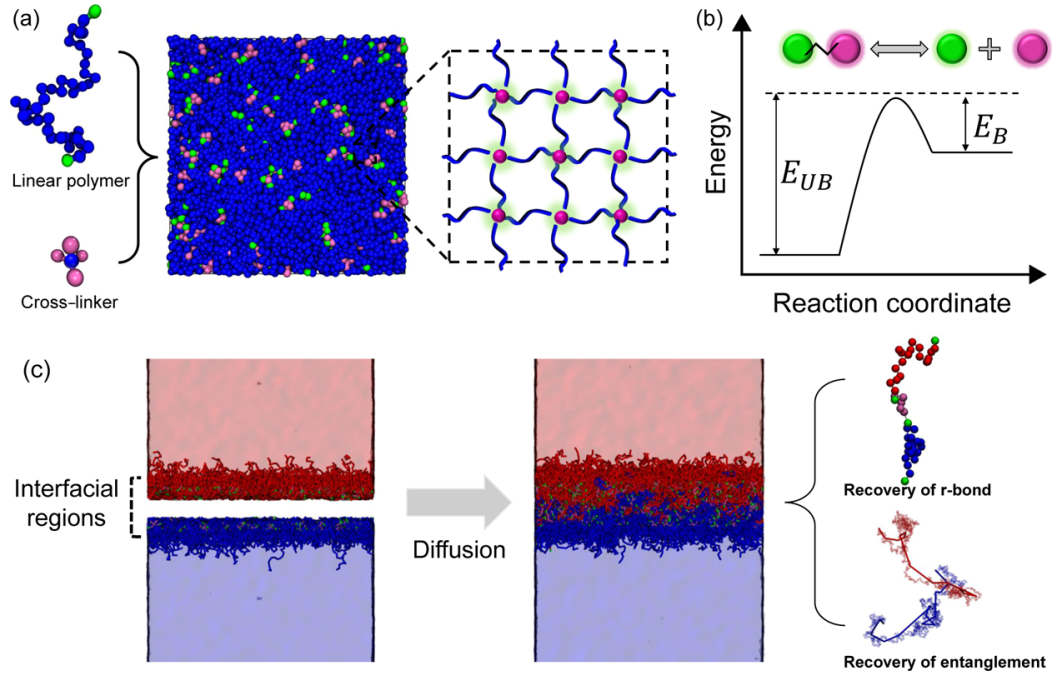

Figure 1. Simulation models of supramolecular polymer networks. (a) Supramolecular polymer network is formed by the linear polymer chains and cross-linkers. A linear polymer chain has two sticky ends, colored green. A cross-linker is a star molecule with four sticky ends (functionality $\phi=4$, colored pink) connected by a central bead. The zoom-in figure is a sketch showing the ideal dynamic network. (b) Reaction of r-bonds between sticky ends are governed by the binding energy $E_{\mathrm{B}}$ and unbinding energy $E_{\mathrm{UB}}$ during a $\mathrm{MC}$ step. The green bead represents the sticky end from the linear polymer chain. The pink one is the sticky end from the cross-linker. (c) Illustration of the self-adhesion process of the fractured supramolecular polymer network. The upper and lower sides of the fractured interface is colored with red and blue with transparency, respectively. Those polymers in the interfacial region are highlighted. The fractured interface is self-healed as the healing time increases. At the healed interface, both the r-bond and entanglements (entangled systems only) are recovered.

process at room temperature. The more extensively reported characteristic healing times exceed $24 \mathrm{~h},{ }^{16}$ such as polymer networks cross-linked by metal-ligand interactions ${ }^{8}$ and reversible Diels-Alder bonds. ${ }^{14}$ Therefore, understanding the self-healing mechanisms behind the diverse healing times is essential for better material design. ${ }^{17-20}$

Despite extensive investigations on the molecular mechanism and equilibrium healing time $\tau_{\text {eq }}$ for supramolecular polymers, widely different and often conflicting results have been reported. The equilibrium healing time $\tau_{\mathrm{eq}}$ is the key feature to characterize the healing efficiency of supramolecular polymers, which denotes the time to fully repair the interfacial strength of a fractured system. For example, Wool and O'Connor ${ }^{21}$ theoretically studied the crack healing of thermoplastic polymers within the framework of the reptation model. $^{22,23}$ They proposed that the full recovery of a crack is achieved at the reptation time $\tau_{\text {rep }}$ when an entire chain escapes from its original tube for molecular diffusion along the polymer-polymer interface. ${ }^{24,25}$ Thus, $\tau_{\text {eq }} \simeq \tau_{\text {rep }}$ and $\sim N^{3}$, as $N$ is the polymerization degree of the polymers. However, molecular dynamics (MD) simulations on the welding ${ }^{26}$ and healing ${ }^{27}$ between polymer-polymer interfaces revealed that the interfacial strength was fully recovered long before chains completely disengaged from their tubes and $\tau_{\text {eq }} \ll \tau_{\text {rep }}{ }^{28}$ Stukalin et al. $^{18}$ provided a comprehensive theoretical framework for the self-healing of unentangled supramolecular polymers. They suggested that equilibrium healing time $\tau_{\text {eq }}$ depends on multiple variables, such as the waiting time, bond lifetime of the r-bond, and relaxation time of the dangling chains at fracture interfaces. Recently, Hornat and Urban ${ }^{20}$ proposed that entropy energy and surface energy might also drive the self-healing of thermoplastic polyurethane fibers. In short, understanding the quantitative relationship between the various chain relaxation timescales and $\tau_{\text {eq }}$ represents an important but yet unsolved problem in the physics of selfhealable supramolecular polymers.
To provide an in-depth understanding of the equilibrium healing time $\tau_{\text {eq }}$ it is necessary to characterize the intrinsic relaxation timescales in a supramolecular polymer network and for the evolution of interfacial molecular structures (IMSs) at the fractured interface during the healing process. ${ }^{18}$ The complexity of polymer dynamics and the difficulty in calibrating the interfacial structures, however, largely hamper the interpretation of the equilibrium healing time $\tau_{\text {eq }}$. On the one hand, the polymer relaxation in a supramolecular polymer network is not only affected by its chain length and topological constraints such as entanglements but also by the dynamics of the r-bond. The relaxation of supramolecular polymers depends on at least four variables: ${ }^{29,30}$ (i) the r-bond strength, (ii) the number, (iii) the distribution of stickers along a precursor polymer chain, and (iv) the chain length of precursor polymers. However, quantitative studies on polymer relaxation are limited by the difficulty in characterizing the r-bond lifetime under the influence of polymer chains and network architecture in experiments. ${ }^{31,32}$ On the other hand, the mechanical strength of a healed fractured interface is determined by the reformations of the r-bond and entanglements that bridge the interfacial gap. ${ }^{18,33-37}$ These microscopic molecular structures are not directly observable in experiments, and the theory is currently limited to the unentangled supramolecular polymers. ${ }^{18}$ The evolution of IMSs remains unknown for entangled supramolecular polymer networks, which is more common in experiments and real applications.

To address these issues, we developed a MD/Monte Carlo (MC) hybrid method to study the self-adhesion of supramolecular polymer networks with equilibrated fractured interfaces. Such a self-adhesion process has an upper limit of self-repair time for fractured supramolecular polymer networks. ${ }^{18}$ The association and dissociation of $r$-bonds is controlled through a MC step ${ }^{38}$ with a binding energy barrier $E_{\mathrm{B}}$ and unbinding energy barrier $E_{\mathrm{UB}}$. The architecture of the 
supramolecular network in our simulations is well controlled, mimicking the four-arm end-functionalized ideal reversible networks ${ }^{2,39}$ (Figure 1). Taking the advantages of MD simulations, the evolution of r-bonds and entanglements can be directly monitored. With such a system at hand, we can calibrate all different intrinsic relaxation timescales, the mechanical response of a fractured system, and the reformation of IMSs for supramolecular polymer networks. We systematically study chain dynamics and the self-adhesion process of both unentangled and entangled supramolecular polymer networks that are cross-linked by the r-bond. The intrinsic relaxation timescales of these polymers are first calibrated under the framework of the sticky Rouse/reptation model . ${ }^{40,41}$ We find that the sticky Rouse time $\tau_{\mathrm{R}}^{\mathrm{s}}$ is largely determined by the lifetime of the r-bond $\tau_{\mathrm{b}}$ for short chains. For long chains, on the other hand, $\tau_{\mathrm{R}}^{\mathrm{s}}$ is greatly influenced by chain entanglements and exceeds $\tau_{\mathrm{b}}$ by far. We then investigate the self-adhesion process of fractured systems with an equilibrated fractured interface. While for unentangled networks, their interfacial strength is found to linearly depend on the areal density of the r-bond across the fractured interface, the interfacial strength of entangled systems is almost linearly correlated with the areal densities of the r-bond and entanglements bridging the fractured interface. When placing the healing time into the context of intrinsic relaxation timescales of supramolecular polymer networks, we find that the sticky Rouse time $\tau_{\mathrm{R}}^{\mathrm{s}}$ features the self-adhesion process of all fractured systems, representing the full recovery of interfacial strength. At this critical timescale, two things happened to guarantee the full recovery of fractured systems: (i) polymer chains have diffused across the fractured interface with a displacement comparable to their sizes; (ii) the crossed stickers and polymer chains have updated their r-bond and entanglements (entangled systems only), respectively.

\section{COMPUTATIONAL MODEL AND METHODS}

Model of the Polymer Chain and Cross-Linker. As shown in Figure 1a, a supramolecular network consists of linear finite-extensible nonlinear elastic (FENE) polymer chains ${ }^{42}$ and cross-linkers. Each polymer chain has two sticky ends. A cross-linker is modeled by a star molecule with four sticky ends, connected with a central bead. r-bonds can only occur between the sticky ends of a polymer chain and a cross-linker. At each instance in time, every sticky end can potentially establish a single r-bond.

The computational model of polymer chains is based on KremerGrest's bead spring model. ${ }^{42}$ All beads interact with each other through the LJ potential

$$
U_{\mathrm{LJ}}(r)=4 \varepsilon\left[\left(\frac{\sigma}{r}\right)^{12}-\left(\frac{\sigma}{r}\right)^{6}\right]-4 \varepsilon\left[\left(\frac{\sigma}{r_{\mathrm{c}}}\right)^{12}-\left(\frac{\sigma}{r_{\mathrm{c}}}\right)^{6}\right]
$$

where $r$ is the distance between two beads. $r_{\mathrm{c}}$ is the cutoff distance with a value of $2.5 \sigma .^{43} \varepsilon$ is the energy unit. $\sigma$ is the bead diameter. An additional unbreakable FENE potential is applied on two connected beads along a polymer chain and within a cross-linker

$$
U_{\mathrm{FENE}}(r)=-\frac{1}{2} k R_{0}^{2} \ln \left[1-\left(r / R_{0}\right)^{2}\right]
$$

where $R_{0}=1.5 \sigma$ and $k=30 k_{\mathrm{B}} / \sigma^{2}$. Each linear polymer chain has $N$ monomers with its two terminal beads (sticky ends). These sticky ends are reactive to form r-bonds with sticky ends of cross-linkers. Each cross-linker is represented by a star molecule consisting of five beads. A central bead is connected with four terminal beads. Each terminal bead in a cross-linker is considered as a sticky end. Every bead in the system has mass $m$. LJ units are used to present the simulation results by setting $\sigma=\varepsilon=m=1$ and the Boltzmann constant $k_{\mathrm{B}}=1 . \tau=\sigma(m / \varepsilon)^{1 / 2}$ is the characteristic time unit.

Model of the Reversible r-Bond. The association and dissociation of the r-bond is modeled as a reversible chemical reaction. The reversible dynamic bonds between sticky candidates in polymer chains and cross-linkers are represented by the reaction potential

$$
U_{\mathrm{RXN}}(r)=\frac{k^{\prime}}{2} \Omega(r, t)\left(r-r_{0}\right)^{2}
$$

where $k^{\prime}$ is the bond strength. $r$ is the distance between two sticky candidates. $r_{0}$ is the equilibrium distance of the r-bond. We set $r_{0}=$ $1.0 \sigma$ to ensure that the averaged length of the r-bond is similar to that of the FENE bond. $\Omega(r, t)$ is a binary function such that $\Omega(r, t)=1$ if a r-bond is established, while $\Omega(r, t)=0$ if the r-bond is broken. The state of $\Omega(r, t)$ is updated every time step $\Delta t$ through a Metropolis $\mathrm{MC}$ algorithm based on the Bell model as follows $38,44-46$

$$
\Omega(r, t)= \begin{cases}1 & \text { if } \Omega(r, t-\Delta t)=0 \text { and } \xi<e^{-\Delta E_{\mathrm{B}} / k_{\mathrm{B}} T} \\ & \text { and } r<r_{\mathrm{rxn}} \\ 0 & \text { if } \Omega(r, t-\Delta t)=1 \text { and } \\ & \xi<e^{-\left(\Delta E_{\mathrm{UB}}-f_{\mathrm{RXN}} l_{0}\right) / k_{\mathrm{B}} T} \\ & \text { otherwise }\end{cases}
$$

where $\xi$ is an equally distributed random number between 0 and 1 generated in every time step. $E_{\mathrm{B}}$ and $E_{\mathrm{UB}}$ are the energy barriers (in unit of $k_{\mathrm{B}} T$ ) for binding and unbinding, respectively. $r_{\mathrm{rxn}}$ is a reaction radius within which a r-bond between the two sticky candidates may be established. $f_{\mathrm{RXN}}$ is the force applied on the r-bond. $l_{0}$ is the characteristic bond length, representing the influence of the applied force on the unbinding probability.

The binding and unbinding energies are set as $E_{\mathrm{B}}=1 k_{\mathrm{B}} T$ and $E_{\mathrm{UB}}=$ $14 k_{\mathrm{B}} T$, respectively. $E_{\mathrm{B}}=1 k_{\mathrm{B}} T$ is chosen to ensure a fast bond formation process. ${ }^{38}$ The choice of $E_{\mathrm{UB}}=14 k_{\mathrm{B}} T$ is based on the balance of three factors: (1) a typical r-bond strength is on the order of a few to tens of $k_{\mathrm{B}} T_{;}^{31}$ (2) the maximum stress of supramolecular polymer networks in simulations should be large enough to identify the difference of fractured networks at different healing times. A larger value of $E_{\mathrm{UB}}$ results in a larger maximum stress value during mechanical tests; (3) the fully recovered state of a fractured system can be observed in simulations. A larger value of $E_{\mathrm{UB}}$ also results in a longer healing process. The choices of the bond strength $k$, reaction radius $r_{\text {rxn }}$, and characteristic bond length $l_{0}$ are based on the factor that the force caused by the topological change of the r-bond is small enough compared to that of a covalent bond. We have $k^{\prime}=100 \varepsilon, r_{\mathrm{rxn}}$ $=1.1 \sigma$, and $l_{0}=0.1 \sigma$, as suggested in ref 38 . Under such energy barriers, over $95 \%$ of sticky ends are bound with their partners at each time step.

Simulation Protocol. To systematically investigate the influence of the polymer chain length (or polymerization degree) on the selfadhesion of supramolecular networks, we have seven different systems in total, that is, $N=20,30,40,60,100,200$, and 500. Each system has polymer beads around 100,000. Correspondingly, the number of polymer chains $n_{\mathrm{ch}}$ in the established system are 5000, 3334, 2500, $1668,1000,500$, and 200 , respectively. To obtain an ideal reversible polymer network, the number of cross-linker $n_{\text {eff }}$ added in the corresponding system is $n_{\text {eff }}=n_{\mathrm{ch}} / 2$. As indicated by the entanglement analysis of the $\mathrm{Z1}$ code $^{47}$ and our previous studies, ${ }^{48-51}$ the entanglement length of these polymer chains is around $N_{\mathrm{e}} \sim 40$. Therefore, the entire seven systems can be classified into two categories: the polymer chains are unentangled and entangled for $N \leq$ 40 and $N \geq 60$, respectively. The time step used in all simulations was $\Delta t=0.01 \tau$. All the simulations were performed by using the largescale atomic/molecular massively parallel simulator (LAMMPS) package ${ }_{53}^{52}$ The snapshots were generated by the visual MD software 

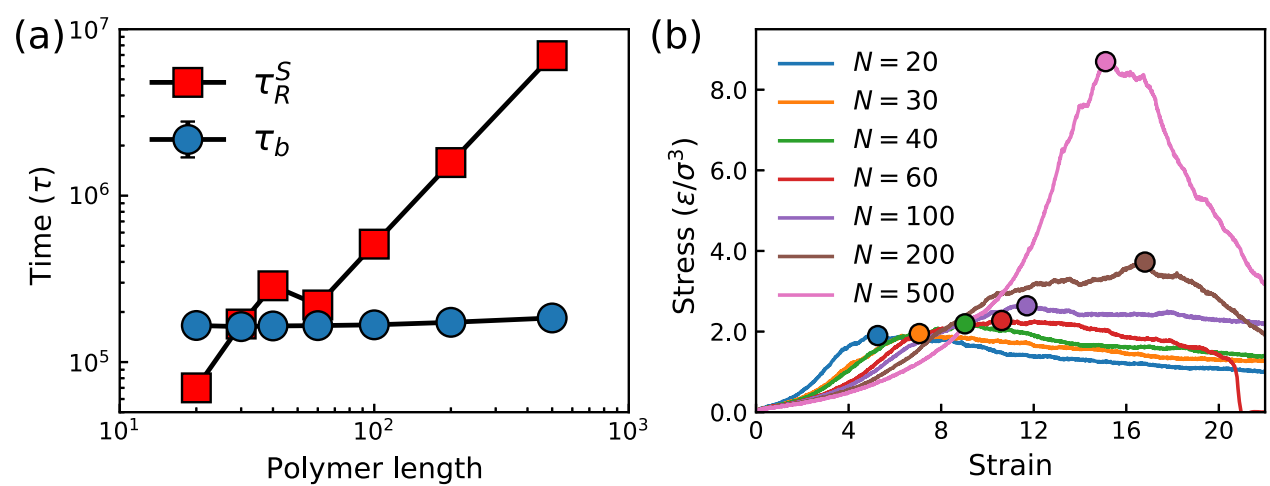

Figure 2. Calibration of bulk supramolecular polymer networks. (a) Characteristic timescales of supramolecular polymer networks. The lifetime of dynamic bonds $\tau_{\mathrm{b}}$ and sticky Rouse time $\tau_{\mathrm{R}}^{\mathrm{s}}$ are plotted against the linear polymer chain length $N$. (b) Stress-strain curves of the supramolecular polymer networks under uniaxial tension. The marked dot in each curve denotes the maximum stress $\sigma_{\max }$ during the tension.

We have developed a proper simulation protocol for each system to systematically investigate the properties of supramolecular polymer networks, including dynamic behavior, mechanical performance, and self-healing ability. The protocol for each system consists of five steps as follows:

Step 1: Initialization. Pre-equilibrated initial configurations were created by using our hybrid $\mathrm{MD} / \mathrm{MC}$ method. ${ }^{54}$ In this method, thin polymer chains are first randomly placed in the simulation box. The volume of each bead in a polymer chain is then increased by using an adaptive time step and time-dependent potentials. We slightly modified this algorithm to randomly place the star molecules (cross-linkers). Subsequently, these pre-equilibrated configurations were further relaxed through the bond swap algorithm (BSA). ${ }^{55}$ The BSA attempts to change a portion of one chain with that of another chain without changing the length of each polymer chain. It allows the effective relaxation of polymer configurations. In the BSA process, the temperature was held constant $(T=1.0)$ by a Langevin thermostat with a damping constant $\Gamma=0.5 \tau^{-1}$. $^{42}$ The reaction potential in eq 3 was not introduced during the entire process of step 1 . The sticky ends in both polymer chains and cross-linkers were treated as the beads in the main part of a polymer chain.

Step 2: Relaxation of Bulk Systems. After step 1, the reaction potential in (3) was then initiated for each system to establish a polymer network. In this step, the temperature and pressure of a system are controlled at $T=1.0$ and $P=0$, respectively. After simulation time around $10^{6} \tau$, the number of $\mathrm{r}$-bonds between sticky candidates is saturated. In addition, the volume of each system also reaches a constant value with slight fluctuation. Both of these indicate the fully relaxed state of the polymer networks. The bead number density of each system was the same as previous results with a value of $\rho=0.89 / \sigma^{-3} .{ }^{42,43}$ After full relaxation, each system was further relaxed up to $10^{7} \tau$ to obtain the dynamic properties of polymer chains and the r-bond for bulk systems. At an equilibrated system, the averaged rbond number is unchanging over time, that is, the association and dissociation processes are under dynamic equilibrium. Please see the averaged bound sticky ends in a cross-linker $\langle\phi\rangle$ in Table S1 of the Supporting Information for details about each system.

Step 3: "Ideal Cut" to Introduce a Fractured Interface. To create a fractured interface, an "ideal cut" was introduced for each system along the $x y$-plane at $z=0$. The "ideal cut" breaks the reversible dynamic bonds of dangling chains, which are at the opposite sides of the cutting surface. These dangling chains were then pushed to their own sides by using a confining potential

$$
U_{\text {conf }}(z)=0.01 k_{\mathrm{B}} T \frac{|z|}{\sigma}
$$

with $-L_{z} / 2 \leq z \geq L_{z} / 2 . L_{z}$ is the size of the simulation box along the $z$ direction. During the "ideal cut", the MC update of the r-bond was not carried out.

After a fractured interface was created, a waiting stage was introduced to exclude the influence of dangling chains during the self- adhesion process. The dangling chains with open sticky ends can quickly diffuse across the fractured interface and find sticky candidates on the other side during the tension process (especially for the short chains). Therefore, we cannot determine the exact healing time during the self-adhesion process. To avoid this scenario, in the waiting stage, the MC update for the r-bond is introduced again. Simultaneously, a wall potential is applied at $z=0$

$$
U_{\text {wall }}(z)=4 \varepsilon\left[\left(\frac{\sigma}{z}\right)^{12}-\left(\frac{\sigma}{z}\right)^{6}\right], \quad|z|<2^{1 / 6} \sigma
$$

The polymer chains near the fractured surface were not allowed to interact with each other over the fractured interface. The waiting period lasts for $10^{6} \tau$, which is long enough to relax the dangling chains. During the waiting period, the open sticky ends in dangling chains are able to form new r-bonds at their own sides. Therefore, the self-adhesion process in our simulations has an upper limit of selfrepair time for fractured supramolecular polymer networks. ${ }^{18}$ After the waiting process, the equilibrated fractured systems have the same averaged bound sticky ends in each cross-linker $\langle\phi\rangle$ as the corresponding bulk systems given in Table S1.

Step 4: Self-Adhesion Process. After step 3, each fractured system was then relaxed with an NPT ensemble $(T=1.0$ and $P=0)$ to heal the fractured interface. The wall potential in eq 6 was removed during this self-adhesion process. With the increment of healing time, the polymer chains at the fractured interface diffuse across the interfacial gap and penetrate into each other. The sticky ends of these polymer chains are able to find a new partner on the other side of the fractured surface. The polymer chains with two bound sticky ends at each side of the fractured interface form a bridge, which can carry the interfacial stress. For entangled polymer systems, these polymer chains are able to form new entanglements with the polymers that originally locate on the other side of the fractured interface. These newly established entanglements across the interface can also form bridges to increase the interfacial strength. The total simulation time for the self-adhesion process can be up to $10^{7} \tau$ depending on the polymerization degree of polymer chains.

Step 5: Uniaxial Tension Process. A uniaxial tension process was applied to determine the interfacial strength of a fractured system after a certain healing time. During the uniaxial tension process, the temperature was controlled at $T=1.0$. The pressure in $x$ and $y$ directions was maintained at $P=0$. A tested system was deformed gradually along the $z$ direction (perpendicular to the fractured interface) with a strain rate $\dot{\varepsilon}$. It is not easy to choose a suitable strain rate. If the strain rate $\dot{\varepsilon}$ is too small, the fractured system will automatically heal during the uniaxial tension process. Therefore, we cannot differentiate it from the self-adhesion process in step 4 . To minimize its influence during the uniaxial tension process, a reduced temperature was applied in ref 26 for the pure entangled polymer systems after welding. However, the reaction behavior of the r-bond will be changed if we use a reduced temperature. On the other hand, if 

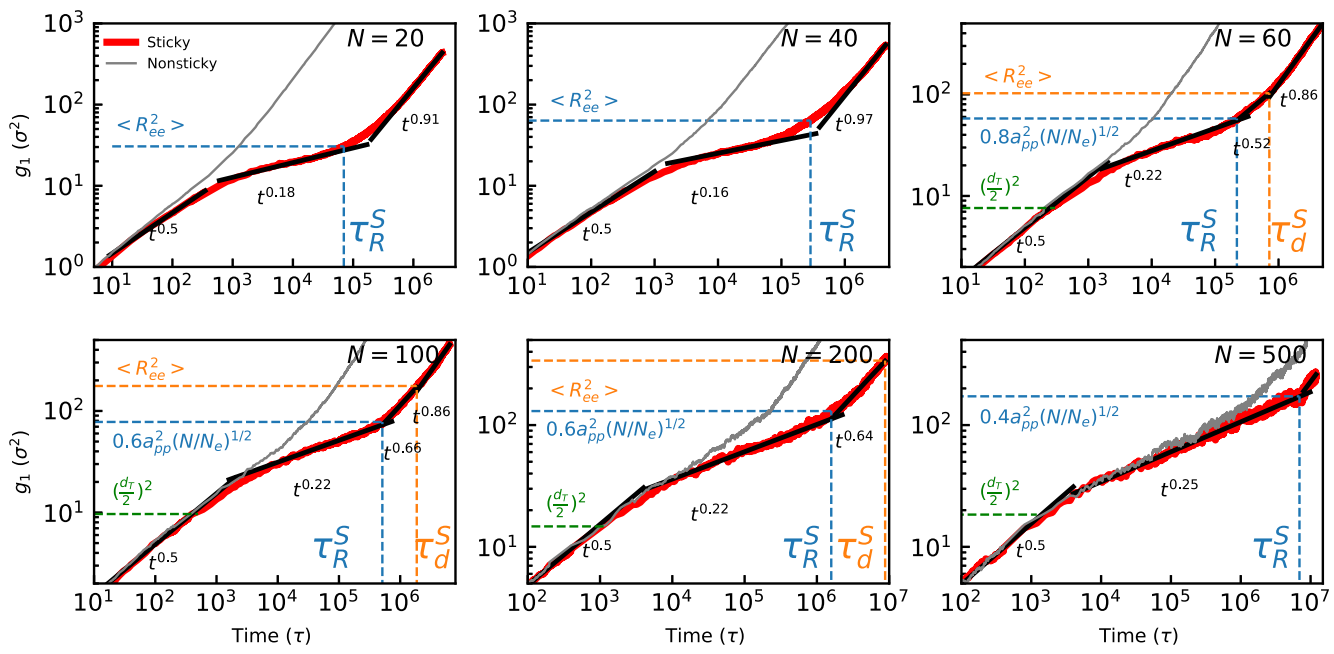

Figure 3. Mean squared displacement of inner monomers $g_{1}(t)$ for different polymer chain lengths. Both the pure polymer melts (the curves in gray) and polymer networks with reversible dynamic bonds (the curves in red) are given. To differentiate time regions, the sticky Rouse time $\tau_{\mathrm{R}}^{\mathrm{s}}$ and sticky disentanglement time $\tau_{\mathrm{d}}^{s}$ are marked in these figures. The corresponding displacements at $t=\tau_{\mathrm{R}}^{s}$ and $t=\tau_{\mathrm{d}}^{s}$ are also presented. In addition, the scaling laws at different time regions are marked in each figure.

Table 1. Summary of Structural and Mechanical Properties of the Bulk Systems ${ }^{a}$

\begin{tabular}{|c|c|c|c|c|c|c|c|c|c|c|c|c|}
\hline$N$ & $\langle Z\rangle$ & $\left\langle N_{\mathrm{e}}\right\rangle$ & $\langle\phi\rangle$ & $\begin{array}{c}\left\langle R_{\mathrm{ee}}^{2}\right\rangle^{1 / 2} \\
(\sigma)\end{array}$ & $\begin{array}{c}\sigma_{\max }^{\text {bulk }} \\
\left(\varepsilon \sigma^{-3}\right)\end{array}$ & $\begin{array}{c}\rho_{\text {eff }}^{\text {bulk }} \\
\left(10^{-2} \sigma^{-2}\right)\end{array}$ & $\begin{array}{c}\rho_{\text {ent }}^{\text {bulk }} \\
\left(10^{-2} \sigma^{-2}\right)\end{array}$ & $\begin{array}{c}\tau_{\mathrm{b}} \\
\left(10^{5} \tau\right)\end{array}$ & $\begin{array}{c}\tau_{\mathrm{R}}^{\mathrm{s}} \\
\left(10^{5} \tau\right)\end{array}$ & $\begin{array}{c}\tau_{\mathrm{d}}^{\mathrm{s}} \\
\left(10^{5} \tau\right)\end{array}$ & $\begin{array}{c}\left\langle\mathrm{a}_{\mathrm{pp}}\right\rangle \\
(\sigma)\end{array}$ & $\begin{array}{l}d_{\mathrm{T}} \\
(\sigma)\end{array}$ \\
\hline 20 & N/A & N/A & 3.95 & 5.53 & 1.95 & 9.93 & N/A & 1.66 & 0.70 & N/A & N/A & N/A \\
\hline 30 & $\mathrm{~N} / \mathrm{A}$ & N/A & 3.94 & 6.94 & 1.98 & 8.73 & $\mathrm{~N} / \mathrm{A}$ & 1.63 & 1.69 & N/A & $\mathrm{N} / \mathrm{A}$ & $\mathrm{N} / \mathrm{A}$ \\
\hline 40 & $\mathrm{~N} / \mathrm{A}$ & N/A & 3.94 & 8.15 & 2.15 & 7.61 & N/A & 1.65 & 2.88 & N/A & N/A & N/A \\
\hline 60 & 1.48 & 40.46 & 3.93 & 10.13 & 2.35 & 6.30 & 1.27 & 1.66 & 2.20 & 7.16 & 7.80 & 5.52 \\
\hline 100 & 2.69 & 37.16 & 3.92 & 13.27 & 2.70 & 5.21 & 2.07 & 1.67 & 5.11 & 18.66 & 8.61 & 6.23 \\
\hline 200 & 4.98 & 40.19 & 3.85 & 18.40 & 3.75 & 3.65 & 2.87 & 1.73 & 15.80 & 87.93 & 10.07 & 7.68 \\
\hline 500 & 11.08 & 45.13 & 3.83 & 29.08 & 9.33 & 2.27 & 3.42 & 1.84 & 69.37 & & 11.85 & 8.58 \\
\hline
\end{tabular}

${ }^{a}$ Number of monomers for each polymer chain, $N$. Entanglement length, $\left\langle N_{e}\right\rangle$ and number of entanglement per chain, $\langle Z\rangle$ obtained from primitive path analysis of $\mathrm{Z} 1$ code, defined by the kink assumption. The averaged bound sticky ends in a cross-linker, $\langle\phi\rangle$. End-to-end distance, $\left\langle R_{\mathrm{ee}}^{2}\right\rangle^{1 / 2}$. The

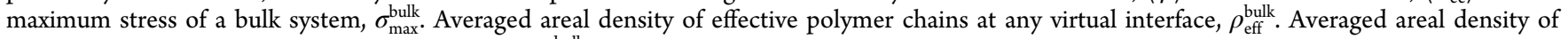
effective entangled strands at any virtual interface, $\rho_{\text {ent }}^{\text {bul }}$. The averaged bond lifetime $\tau_{b}$. The sticky Rouse time $\tau_{\mathrm{R}}^{\mathrm{s}}$. The sticky disentanglement time, $\tau_{\mathrm{d}}^{\mathrm{s}}$. The primitive path step length, $\left\langle a_{\mathrm{pp}}\right\rangle$. The tube diameter, $d_{\mathrm{T}}$.

the strain rate is too large, a physically unrealistic stretched covalent bond might be introduced. As a balance, we used a strain rate of $\dot{\varepsilon}=$ $10^{-3} / \tau$, at which a reasonable mechanical response of the KremerGrest model can be reflected. ${ }^{77}$ To analyze the mechanical behavior of bulk systems, the same uniaxial tension process was applied to the fully relaxed systems after step 2. The stress during the uniaxial extension tests is calculated based on the well-known IrvingKirkwood formula ${ }^{56}$

$$
\sigma_{i j}=-\left\langle\frac{1}{V} \sum_{k=1}^{N}\left(m_{k} v_{k, i} v_{k, j}+r_{k, i} F_{k, j}\right)\right\rangle
$$

where $V$ and $N$ are the total volume and bead numbers in the system, respectively. $m_{k}, v_{k, i}, r_{k, i}$, and $F_{k, i}$ are the mass, velocity, position, and total force, respectively, on the bead $k . i$ and $j$ can be taken as $x, y$, and $z$ directions. We take the normal stress in the tension direction for stress-strain curves.

\section{RESULTS}

Calibration of Bulk Supramolecular Polymers. We first calibrate the intrinsic timescales and mechanical properties of these bulk supramolecular polymer networks. To calibrate the dynamic properties of bulk systems, each system first experiences a long relaxation period at constant temperature $T=1.0 \varepsilon / k_{\mathrm{B}} T$ and pressure $P=0$. There are two different timescales for supramolecular networks, that is, the lifetime of r-bond $\tau_{\mathrm{b}}$ and the relaxation time of the polymer network. $\tau_{\mathrm{b}}$ is the average time that two stickers spend the bonded state before the first successful break. ${ }^{18}$ In our simulations, we monitored lifetime of each r-bond in the bulk systems. The histogram distribution of lifetime is then fitted by an exponential function $f(t)=A_{0} \exp \left(-t / \tau_{\mathrm{b}}\right)$. The $\tau_{\mathrm{b}}$ values extracted through the fitted functions are the ones presented in Figure 2 (see Figure $S 1$ in the Supporting Information for details). $\tau_{b}$ only slightly increases with the chain length $N$ and remains around a characteristic value of $\tau_{\mathrm{b}} \approx 1.7 \times 10^{5} \tau$. It is worth to note that the precise measurements of a bond lifetime within a supramolecular network are unavailable in experiments. In experiments, the bond lifetime is often approximated in the dilute solutions, ${ }^{57,58}$ while the influence of the polymer connectivity remains unknown.$^{32}$ This also makes our simulation system a unique candidate to examine all the intrinsic timescales of a supramolecular network.

We adopt the mean squared displacement (MSD) of monomers to characterize the diffusion behavior of polymer chains with sticky ends. Particularly, the sticky Rouse time $\tau_{\mathrm{R}}^{\mathrm{s}}$ and sticky disentanglement time $\tau_{\mathrm{d}}^{\mathrm{s}}$ are discussed in detail. The MSD of a monomer $g_{1}(t)$ is defined as 


$$
g_{1}(t)=\frac{1}{N} \sum_{i=1}^{N}\left\langle\left[\vec{r}_{i}(t)-\vec{r}_{i}(0)\right]^{2}\right\rangle
$$

with $\vec{r}_{i}(t)$ denoting the position of a monomer at time $t$. The inner five monomers in a polymer chain are used to calculate the $g_{1}(t)$ to exclude the influence of terminal beads. The MSD curves of unentangled sticky polymers can be classified into three different time regions (see Figure 3): (1) at the initial diffusive time region, the polymer chains cannot the feel the constraints from the r-bond at sticky ends and behave as Rouse-like chains with a scaling law of $g_{1}(t) \propto t^{1 / 2}$; (2) after a certain time period, the motion of monomers is slowed down due to sticky ends. At this time region, a scaling law $g_{1}(t) \propto t^{\alpha_{1}}$ with $\alpha_{1}<0.5$ is observed; (3) at the time around $\tau_{\mathrm{b}}\left(\tau_{\mathrm{b}}=1.66\right.$ $\times 10^{5} \tau$ and $1.65 \times 10^{5} \tau$ for $N=20$ and $N=40$, respectively), the motion of monomers speeds up because most of the bound sticky ends are dissociated. The polymer chains are then quickly fully relaxed with a scaling law of $g_{1}(t) \propto t^{\alpha_{2}}\left(\alpha_{2} \sim 1\right)$. We estimate the sticky Rouse time as the time when a monomer diffuses over a distance of its own size $\left\langle R_{\mathrm{ee}}^{2}\right\rangle^{1 / 2}$. As indicated in the crossing point between $\left\langle R_{\mathrm{ee}}^{2}\right\rangle^{1 / 2}$ and MSD of nonsticky polymers in Figure 3, the Rouse time of nonsticky polymers (i.e., pure polymer melts) is much smaller than the bond lifetime. The sticky Rouse time (crossing point between $\left\langle R_{\mathrm{ee}}^{2}\right\rangle^{1 / 2}$ and MSD of sticky polymers) is comparable to the bond lifetime (see Table 1).

Following the concept of the reptation model, the topological constraints from entanglements on a given polymer chain confine its motion into a tube-like region. The tube-like region has a tube diameter $d_{\mathrm{T}}$. The axis of the tube is the shortest path connecting two ends of the strand with the same topology as the strand itself. This axis of the tube is represented by the primitive chain. The primitive chains follow Rouse-like diffusion with a step length of $a_{\mathrm{pp}}$. As given in Figure 3, the MSD curves of entangled sticky polymer chains can be classified into four different regions: (1) When the motion time is smaller than the entanglement time, that is, $t<$ $\tau_{\mathrm{e}}$, the monomers of polymers cannot feel the constraints from tube and r-bonds, as evidenced by the same $g_{1}(t)$ values for sticky and nonsticky polymers. Therefore, the polymer chains behave as Rouse-like chains and follow the scaling law as $g_{1}(t)$ $\propto t^{1 / 2}$; (2) when $\tau_{e}<t<\tau_{\mathrm{R}}^{\mathrm{s}}\left(\tau_{\mathrm{R}}^{\mathrm{s}}\right.$ is the sticky Rouse time), the motion of monomers is influenced by tube constraints and reversible dynamic bonds. The $g_{1}(t)$ follows a scaling law as $g_{1}(t) \propto t^{\beta_{1}}\left(\beta_{1} \sim 0.25\right)$. By comparing the MSD curves of sticky and nonsticky polymers, we find that the r-bonds at the sticky ends largely extend the Rouse time region; (3) when $\tau_{\mathrm{R}}^{\mathrm{s}}<t<$ $\tau_{\mathrm{d}}^{\mathrm{s}}\left(\tau_{\mathrm{d}}^{\mathrm{s}}\right.$ is the sticky disentanglement time $)$, the $g_{1}(t)$ follows a scaling law as $g_{1}(t) \propto t^{\beta_{2}}\left(\beta_{2} \sim 0.5\right)$; (4) when $t>\tau_{\mathrm{d}}^{\mathrm{s}}$, the polymer chains totally escape from original tube constraints. In addition, the r-bonds at sticky ends are already broken at this time region. The polymer chains are fully relaxed with a scaling law of $g_{1}(t) \propto t^{\beta_{3}}\left(\beta_{3} \sim 1\right)$. The sticky disentanglement time $\tau_{\mathrm{d}}^{\mathrm{s}}$ is estimated as the time when a monomer diffuse over a distance of its own size $\left\langle R_{\mathrm{ee}}^{2}\right\rangle^{1 / 2}$. Due to the extremely long relaxation time for $N=500$, we do not have an estimation of $\tau_{\mathrm{d}}^{\mathrm{s}}$ for $N=500$. The $\tau_{\mathrm{R}}^{\mathrm{s}}$ is estimated as the time when the second time region ends. To confirm that the estimated $\tau_{\mathrm{R}}^{\mathrm{s}}$ values are reasonable, we calculate the MSD values at $t=\tau_{\mathrm{R}}^{\mathrm{s}}$. It is found that the MSD values at $t=\tau_{\mathrm{R}}^{\mathrm{s}}$ are comparable to $a_{\mathrm{pp}}^{2}\left(N / N_{\mathrm{e}}\right)^{1 / 2}$. $R_{\mathrm{ee}}, a_{\mathrm{pp}}$, and $N_{\mathrm{e}}$ are the end-to-end distance, primitive path step length, and entanglement length (Supporting Information
Section 2 for detailed information). $a_{\mathrm{pp}}=\left\langle R_{\mathrm{ee}}^{2}\right\rangle /\left\langle L_{\mathrm{pp}}\right\rangle$ is the step length of primitive path form $\mathrm{Z} 1$ analysis, where $\left\langle L_{\mathrm{pp}}\right\rangle$ is the contour length of primitive path. Therefore, we are confident that the estimated $\tau_{\mathrm{R}}^{\mathrm{s}}$ values are reasonable. Furthermore, the sticky disentanglement time $\tau_{\mathrm{d}}^{\mathrm{s}}$ can be correlated with sticky Rouse time $\tau_{\mathrm{R}}^{\mathrm{s}}$ as $\tau_{\mathrm{d}}^{\mathrm{s}}=\omega \mathrm{Z} \tau_{\mathrm{R}}^{\mathrm{s}}$, where $0.6<$ $\omega<2.2$ based on the values in Table 1 .

In short, the relaxation timescales of supramolecular polymers are quantified under the framework of the sticky Rouse/reptation model. The sticky Rouse and sticky reptation models are the most well-established theories for sticky chain dynamics. $^{40,41}$ These models originally focused on strong $r$ bonds and were later extended to weak r-bond by including slip-link models. ${ }^{59}$ The sticky Rouse time $\tau_{\mathrm{R}}^{\mathrm{s}}$ of polymer chains is inferred by the MSD of monomers. Here, we pay close attention on the sticky Rouse time $\tau_{\mathrm{R}}^{\mathrm{s}}$, which will be used as a reference for the self-adhesion time for both the unentangled and entangled systems. At the Rouse timescale $\tau_{\mathrm{R}}^{\mathrm{s}}$, a polymer chain already moves a distance comparable to its size at $\tau_{\mathrm{R}}^{\mathrm{s}}$, that is, a distance on the order of $\left\langle R_{\mathrm{ee}}^{2}\right\rangle^{0.5}$ and for unentangled and entangled polymers, respectively. As shown in Figure $2 a$, the polymer relaxation is determined by the r-bond at sticky ends for short chains $(N=20,30,40)$. Their $\tau_{\mathrm{R}}^{\mathrm{s}}$ values are comparable to the bond lifetime $\tau_{\mathrm{b}}$ on the order of $10^{5} \tau$. In contrast, the dynamic behavior of long chains is constrained by both the topological constraints from entanglements and rbonds. Their $\tau_{\mathrm{R}}^{\mathrm{s}}$ values are much larger than $\tau_{\mathrm{b}}$, on the order of $10^{6} \tau$ for $N=200$. Our simulation results are consistent with the experimental works: (a) if $\tau_{\mathrm{b}}$ is large and well separated from relaxation time of the nonsticky precursor polymer chains $\tau_{\text {relax }}$ the relaxation of sticky chains is governed by the kinetics of r-bond exchange; ${ }^{58,60,61}$ (b) otherwise, the entanglements outweigh the influence of the r-bond and determine the sticky chain dynamics. ${ }^{29,62,63}$

The mechanical properties of bulk systems are tested through uniaxial tension at a strain rate of $\dot{\varepsilon}=10^{-3} / \tau$. As given in Figure $2 b$, the stress-strain curves follow a similar trend for each chain length. The bulk (normal) stress $\sigma_{\text {bulk }}$ first increases with the increment of strain and reaches a maximum value $\sigma_{\max }^{\text {bulk }}$, after which it drops due to the failure of the bulk system. The maximum stress of the unentangled systems just slightly increases from $\sigma_{\max }^{\text {bulk }}=1.95 \varepsilon \sigma^{-3}$ at $N=20$ to $\sigma_{\max }^{\text {bulk }}=$ $2.15 \varepsilon \sigma^{-3}$ at $N=40$. In comparison, $\sigma_{\max }^{\text {bulk }}$ values grow dramatically with increasing chain length for the entangled system after $N=100$. The maximum stress during the uniaxial tension for $N=500$ can reach $\sigma_{\max }^{\text {bulk }}=9.33 \varepsilon \sigma^{-3}$, which is around four times larger than that of $N=20$. This increment of $\sigma_{\max }^{\text {bulk }}$ for the entangled networks indicates the contribution of entanglements to the mechanical strength. ${ }^{51}$ These maximum stress values of bulk systems are later used as references to determine the recovery of interfacial strength for fractured systems. We also calculate the areal densities of effective polymer chains and entangled strands at virtual interfaces of bulk systems as references to compare with those of the fractured interface during the self-healing process. Specifically, we divide a bulk material into 11 slabs with 10 virtual interfaces uniformly. For each virtual interface, the $\rho_{\text {eff }}$ and $\rho_{\text {ent }}$ can be quantified. The averaged values of $\rho_{\text {eff }}^{\text {bulk }}$ and $\rho_{\text {ent }}^{\text {bulk }}$ are listed in Table 1.

Self-Adhesion of Unentangled Supramolecular Polymers. We went on to investigate the self-adhesion of unentangled systems. To create a fractured interface, an "ideal cut" is introduced for each relaxed bulk system to create 
(a)

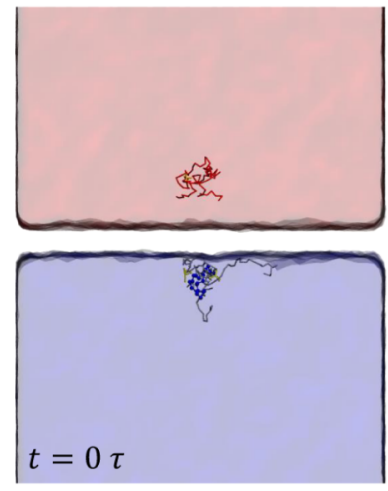

(b)

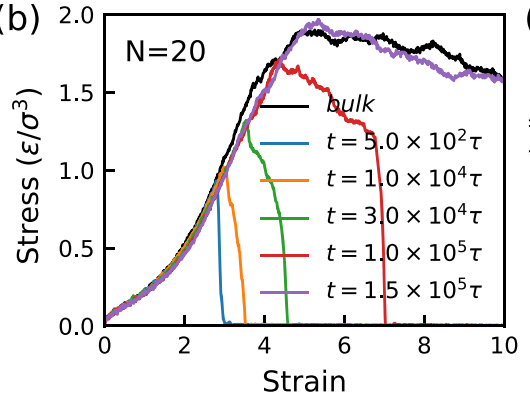

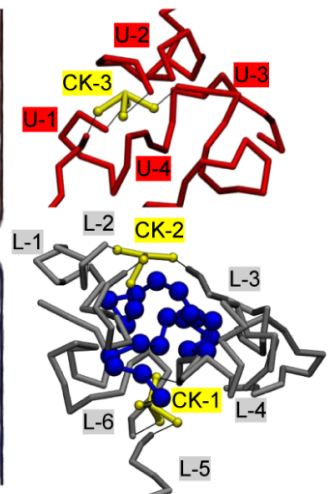

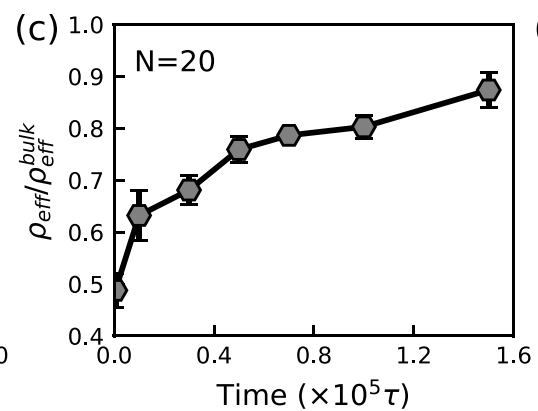

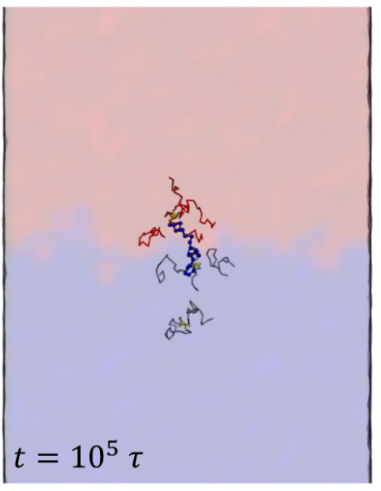

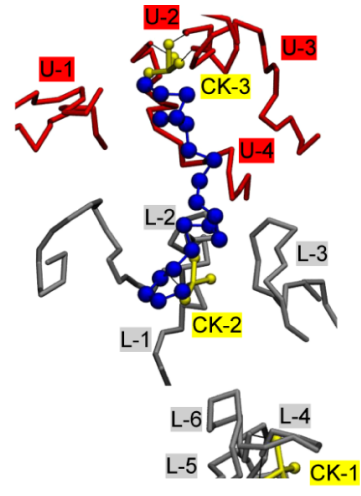

Figure 4. Self-adhesion of unentangled supramolecular polymer networks. (a) Snapshots of the fractured interface during the healing process. The evolution about the formation of an effective polymer chain across the interface is highlighted. The upper and lower sides of the fractured interface are colored with red and blue with transparency, respectively. The effective chain is colored in blue. The involved cross-linkers are colored in yellow. The polymers in the lower and upper side of the interface are colored in silver and red, respectively. (b) Stress-strain curves for the fractured system with $N=20$ and different healing times. (c) Function of normalized areal density of effective polymer chains $\rho_{\text {eff }} / \rho_{\text {eff }}^{\text {bulk }}$ against healing time for $N=20$. (d) Correlation between the normalized areal density of effective polymer chains $\rho_{\text {eff }} / \rho_{\text {eff }}^{\text {bulk }}$ across the fractured interface and normalized maximum recovered stress $\sigma_{\text {rec }} / \sigma_{\text {bulk }}$ for $N=20,30,40$. The error bars of $\rho_{\text {eff }} / \rho_{\text {eff }}^{\text {bulk }}$ and $\sigma_{\text {rec }} / \sigma_{\text {bulk }}$ are obtained from five different samples near a certain healing time and five independent uniaxial tensions at a given healing time, respectively.

an equilibrated fractured interface, as detailed in the previous section. Such a fractured interface has an upper limit of selfrepair time ${ }^{18}$ The fractured system then experiences a healing process at unchanged thermodynamic conditions as the bulk systems. As shown in Figure $4 \mathrm{a}$ for $N=20$, the fractured interface is quickly filled by the polymer chains near the interface during the healing process. The molecular evolution of a polymer chain (colored in blue) that bridges the fractured interface is monitored during the simulation. The blue chain initially locates at the lower side of the interface with its sticky ends bound with the cross-linkers (CK-1 and CK-2) on its own side. These two cross-linkers (CK-1 and CK-2) are also bound with six other polymer chains in the lower side that are marked as (L-1 to L-6) at $t=0 \tau$. As healing time evolves, one sticky end of the blue chain dissociates with its cross-linker and gradually diffuses across the interface. At $t=10^{5} \tau$, the dissociated sticky end of the blue chain has already formed a new bond with a cross-linker (CK-3) that initially locates on the upper side of the interface. Note that the CK-3 was fully bound with four polymer chains (U-1 to U-4) at its own side at $t=0 \tau$. This process observed in our simulation is exactly the "partner exchange" healing mechanism proposed by Rubinstein and co-workers . ${ }^{18}$ These polymers such as the blue chain in the figure can bridge the fractured interface and transfer the load during deformation. ${ }^{18}$ We denote these polymers as effective polymer chains in the present study.

With the help of effective polymer chains, the mechanical strength of the fractured interface is gradually restored. The mechanical properties of the fractured systems are also tested through the same uniaxial tension as the bulk systems. The stress-strain curves at a series of healing times are compared with the corresponding bulk system, as presented in Figure $4 \mathrm{~b}$ for $N=20$. The stress responses of the fractured samples follow the same stress-strain curve as the pristine bulk system but break at a lower $\sigma_{\max }$ value. The $\sigma_{\max }$ of the fractured systems monotonically increases with the healing time. These $\sigma_{\max }$ values are denoted as recovered stress values $\sigma_{\text {rec }}$. Furthermore, the stress-strain curve at $t=1.5 \times 10^{5} \tau$ is almost indistinguishable against the pristine bulk system for $N$ $=20$, indicating full recovery of the interfacial strength. The self-adhesion processes of $N=30$ and $N=40$ follow a similar trend as that of $N=20$, with a fully recovered state around $t=$ $2.0 \times 10^{5} \tau$ (Supporting Information Figure S4).

To establish a direct relationship between the microscopical molecular behaviors and the macroscopic mechanical performance of the fracture systems, we systematically analyze the IMSs. The evolution of the areal density of effective polymer chains $\rho_{\text {eff }}$ at the fractured interface is measured during the entire healing process. We further normalize the $\rho_{\text {eff }}$ by that of the corresponding bulk system $\rho_{\text {eff }}^{\text {bulk }}$. The $\rho_{\text {eff }}^{\text {bulk }}$ is calculated by averaging the effective polymer chains at 10 virtual interfaces of the bulk system. As given in Figure $4 \mathrm{c}$ for $N=20$, the normalized values of $\rho_{\text {eff }} / \rho_{\text {eff }}^{\text {bulk }}$ increase monotonically with the healing duration and reach around $90 \%$ at the time the interfacial strength is fully recovered. More importantly, when we try to correlate between the IMS and its restored mechanical property, the recovered maximum stress values $\sigma_{\text {rec }} / \sigma_{\text {bulk }}$ are almost linearly dependent on $\rho_{\text {eff }} / \rho_{\text {eff }}^{\text {bulk }}$ for all the 
(a)

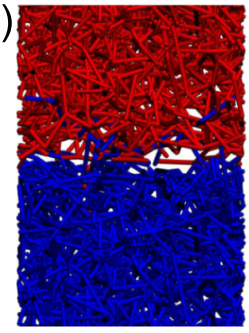

$t=5 \times 10^{3} \tau$

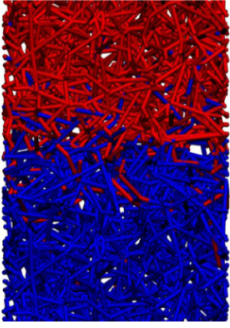

$t=2 \times 10^{6} \tau$

(b)

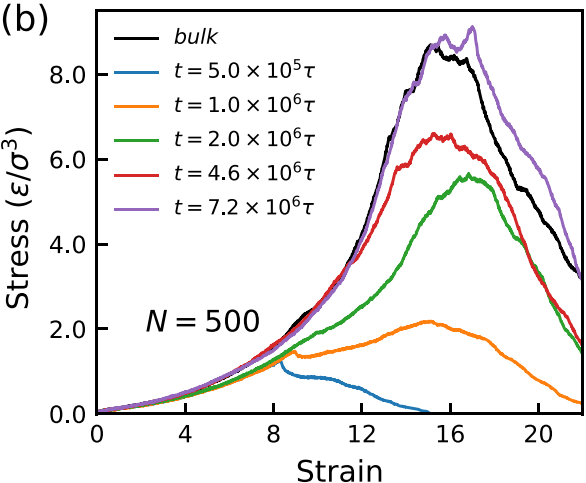

(d)

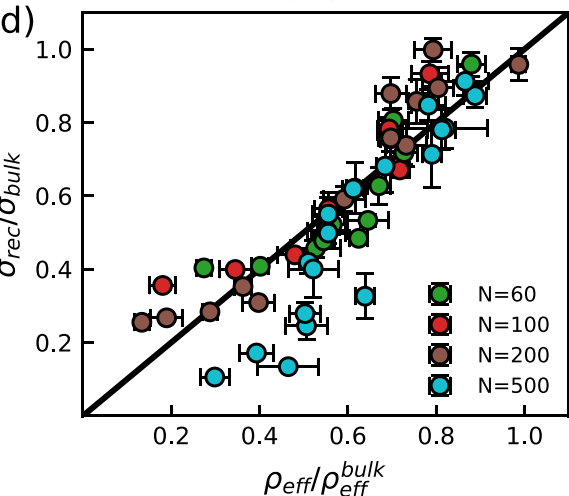

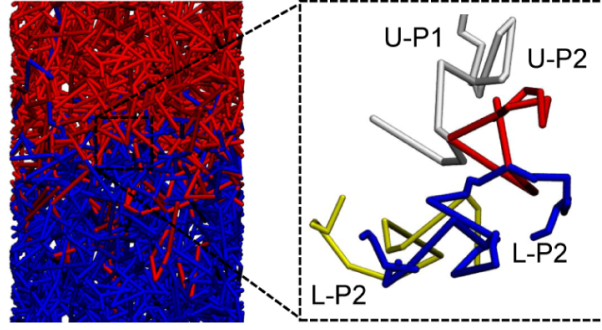

$t=7.2 \times 10^{6} \tau$

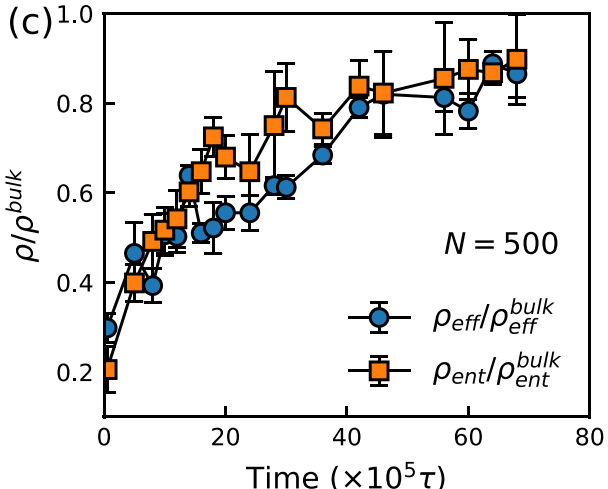

(e)

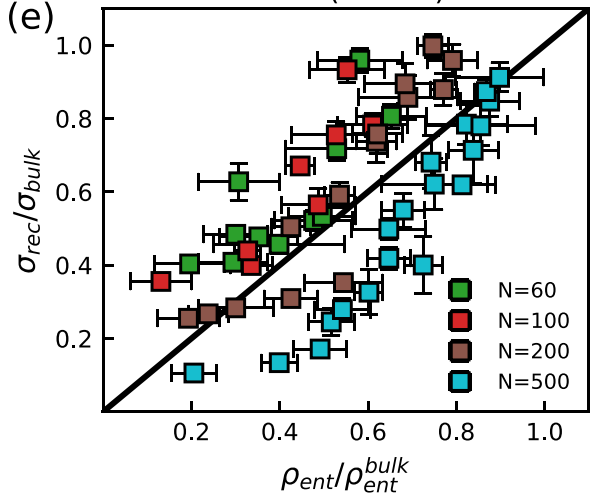

Figure 5. Self-adhesion of entangled supramolecular polymer networks. (a) Snapshots of the primitive path during the healing process. These primitive paths that initially locate the lower and upper sides of the fracture are colored in blue and red, respectively. (b) Stress-strain curves for the fractured system with $N=500$ and different healing times. Note that there is a local maximum at the strain $=8$ in the stress-strain curve of short healing time. This might be related to the initial breaks of the r-bond at the fractured interface. (c) Functions of normalized areal density of effective polymer chains $\rho_{\text {eff }} / \rho_{\text {eff }}^{\text {bulk }}$ and topologically cross-linked (entangled) strands $\rho_{\text {ent }} / \rho_{\text {ent }}^{\text {bulk }}$ against healing time for $N=500$. (d,e) Correlations between normalized maximum recovered stress $\sigma_{\text {rec }} / \sigma_{\text {bulk }}$ and IMSs, such as $\rho_{\text {eff }} / \rho_{\text {eff }}^{\text {bulk }}$ and $\rho_{\text {ent }} / \rho_{\text {ent }}^{\text {bulk }}$.

unentangled systems (Figure 4d). This correlation confirms that the recovered interfacial strength $\sigma_{\text {rec }}$ of the unentangled systems is governed by the areal density of effective polymer chains $\rho_{\text {eff. }}$ We refer to the Supporting Information Figure S4 for the details about the self-adhesion processes of $N=30$ and $N=40$.

Self-Adhesion of Entangled Supramolecular Polymers. We further examine the self-adhesion of entangled systems. The fractured interface takes a long time to recover its mechanical strength for these entangled systems. Figure $5 \mathrm{~b}$ shows the stress-strain curves of the high-molecular-weight $N$ $=500$ systems. The recovery of interfacial strength for $N=500$ follows a similar trend as that of $N=20$. The maximum recovered stress $\sigma_{\text {rec }}$ of the fractured system progressively increases with the increment of healing time. At $t=7.2 \times 10^{6} \tau$ for $N=500$, the interfacial strength is fully restored. The simulation results for other entangled supramolecular polymer networks are provided in Supporting Information Figure S5.
Apart from the restorage of effective polymer chains as for the unentangled systems, the recovery of the entanglements at the fractured interface also contributes to the interfacial strength. ${ }^{26}$ These entanglements at the interface are investigated through the $\mathrm{Z} 1$ code, ${ }^{47}$ as shown in Figure 5a for $N=$ 500. At $t=5 \times 10^{3} \tau$, only a few primitive paths from the upper and lower sides interweave at the interface, while at $t=7.2 \times$ $10^{6} \tau$, the interface is filled with the primitive paths, indicating that the interface already becomes a highly entangled region. At this time, the upper-side entanglement strands such as U-P2 entangled with the strands from the lower side (such as L-P2) at the interface. The strands of U-P2 and L-P2 also entangled with the strands from the upper and lower sides, respectively, at the same time. The entanglement strands, such as U-P2 and L-P2 in the initial fractured gap region, can effectually restore the mechanical strength of the interface.$^{35,36,64}$ We name them as effective entangled strands.

The evolution of the IMS is further systematically inspected to establish its relationship with the recovered interfacial 

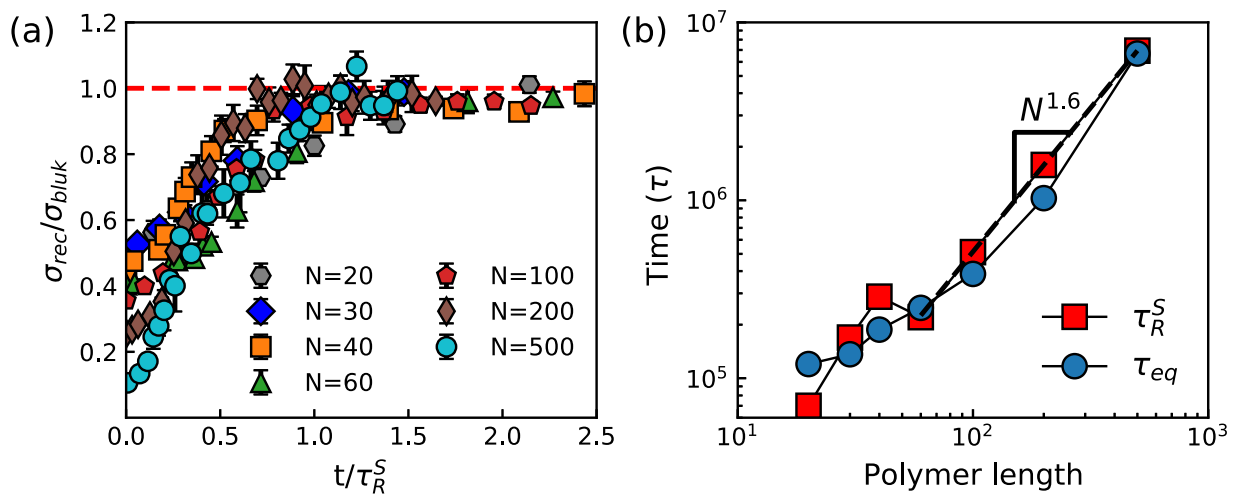

Figure 6. (a) Normalized recovered interfacial strength $\sigma_{\text {rec }} / \sigma_{\text {bulk }}$ against the normalized healing time $t / \tau_{\mathrm{R}}^{\mathrm{s}}$. (b) Comparison between equilibrium healing time $\tau_{\text {eq }}$ and sticky Rouse time $\tau_{\mathrm{R}}^{\mathrm{s}}$, both as a function of polymer chain length $N$.

strength. We calculate both the areal densities of effective polymer chains $\rho_{\text {eff }}$ and topologically cross-linked (entangled) strands $\rho_{\text {ent }}$ at the fractured interface. Both $\rho_{\text {eff }}$ and $\rho_{\text {ent }}$ simultaneously increase with the healing time for $N=500$ (Figure 5c). As given in Supporting Information Figure S5 for more details, the $\rho_{\text {eff }}$ values increase to $>80 \%$ over those of bulk systems for all the entangled systems at the fully recovered state. The $\rho_{\text {ent }}$ values finally recover to $\sim 60 \%$ (for $N=60$ and $N=100$ ) and $>80 \%$ (for $N=200$ and $N=500$ ). This difference indicates that the mechanical contributions of entangled strands for the highly entangled systems might be more significant than that of weakly entangled systems. We further plot the normalized maximum recovered stress against the normalized areal density of effective polymer chains and effective entangled strands, respectively, in Figure 5 d,e. The $\rho_{\text {eff }}$ and $\rho_{\text {ent }}$ almost correlate with the recovered interfacial strength $\sigma_{\text {rec }}$ with a nearly linear relationship. Therefore, we can conclude that the effective polymer chains and effective entangled strands across the fractured interface recover simultaneously and determine the interfacial strength for the healed entangled polymer networks. This observation is consistent with the molecular model for nonlinear elasticity of entangled polymer networks with covalent cross-links. ${ }^{51,65,66}$

Sticky Rouse Time Features the Self-Adhesion Process. Having the evolution of macroscopic interfacial strength and the microscopic IMS at hand, the next step is to evaluate the time needed to fully repair a fractured interface. As shown in Figure 6a, we place the healing process into the context of the intrinsic relaxation timescale of supramolecular polymer networks by normalizing the healing time $t$ with the sticky Rouse time $\tau_{\mathrm{R}}^{\mathrm{s}}$. It is surprising to find that the $\sigma_{\text {rec }} / \sigma_{\text {bulk }}$ of all fractured systems collapse together in terms of normalized healing time $t / \tau_{\mathrm{R}}^{s}$ and almost reach 1.0 (fully recover) at $t=\tau_{\mathrm{R}}^{\mathrm{s}}$. It suggests that the sticky Rouse should be the intrinsic timescale that a supramolecular polymer network needs to fully repair its fractured interface. We further define the equilibrium healing time $\tau_{\text {eq }}$ as the time when the $\sigma_{\text {rec }} / \sigma_{\text {bulk }}$ approaches $90 \%$ (Figure S6) ${ }^{64}$ and compare it with $\tau_{\mathrm{R}}^{\mathrm{s}}$ in Figure $6 \mathrm{~b}$. The $\tau_{\text {eq }}$ values for all chain lengths are comparable to the corresponding $\tau_{\mathrm{R}}^{\mathrm{s}}$. In addition, we can estimate $\tau_{\mathrm{eq}}$ for different chain lengths based on the scaling law between $\tau_{\mathrm{R}}^{\mathrm{s}}$ and $N$. We have $\tau_{\mathrm{R}}^{\mathrm{s}} \propto N^{\alpha}$, with $1<\alpha<2$, which is consistent with the theoretical prediction. ${ }^{67}$ The reasons why the sticky Rouse time features the self-adhesion of supramolecular polymer networks are clarified as follows for unentangled and entangled systems separately.
Since the relaxation time of nonsticky unentangled polymer chains is smaller than the bond lifetime $\tau_{\mathrm{b}}$, the sticky Rouse time $\tau_{\mathrm{R}}^{\mathrm{s}}$ of an unentangled supramolecular polymer network is determined by the r-bond with a value comparable to $\tau_{\mathrm{b}}$ (Figure 2). At the sticky Rouse time, two pivotal things happen: (i) the polymer chains at the fractured interface have diffused across the interfacial gap with a displacement comparable to their sizes; (ii) most sticky ends have broken their original dynamic bonds at $t=0$ and find new sticky partners across the fractured interface. These two aspects ensure that unentangled polymer chains can diffuse across the fractured interface and effectively restore the interfacial strength at $\tau_{\mathrm{R}}^{\mathrm{s}}$.

For entangled systems, both the effective polymer chains and entangled strands contribute to the interfacial strength. ${ }^{51,64}$ The $\tau_{\mathrm{R}}^{\mathrm{s}}$ is significantly larger than the bond lifetime $\tau_{\mathrm{b}}$ (Figure 2a) due to the topological constraints from entanglements. It suggests that most of the original $\mathrm{r}$-bond (at $t=0$ ) are broken at $\tau_{\mathrm{R}}^{\mathrm{s}}$. Furthermore, as we can see in Figure 3, the entangled polymer chains diffuse over a distance of $a_{\mathrm{pp}}^{2}\left(N / N_{\mathrm{e}}\right)^{0.5}$ that is comparable to their sizes. Therefore, the IMS of effective polymer chains can fully restore at $\tau_{\mathrm{R}}^{\mathrm{s}}$ (Figure $5 \mathrm{c}$ ). The only question left is why the effective entangled strands can be fully restored at $t=\tau_{\mathrm{R}}^{\mathrm{s}}$. To further quantify the entanglement evolution for supramolecular polymer networks, we calculate the segment survival probability function $\psi(s, t)$ for bulk systems, ${ }^{68}$ which describes the probability that a primitive segment at a relative contour position $s$ remains in the original tube after time $t$. As presented in Supporting Information Figure S7, at $t=\tau_{\mathrm{R}}^{\mathrm{s}}$, only $20 \%$ of the primitive chain terminals $(s=0,1)$ remained within their original tubes. It suggests that $80 \%$ of the terminal parts of polymer chains already formed new entanglements with other polymer chains at $t=\tau_{\mathrm{R}}^{\mathrm{s}}$. This percentage of $\psi(s, t)$ is consistent with the full recovery state of entangled strands at the fractured interface. Combining these two factors of the diffusive displacement and chance to update their entanglements at $t=\tau_{\mathrm{R}}^{\mathrm{s}}$, we can conclude that the sticky Rouse time is long enough to fully restore the effective entangled strands across the fractured interface.

Discussion. In this work, we studied a subclass of supramolecular networks: telechelic linear chains able to interact with tetrafunctional cross-linkers in the strong association regime (as predefined by the binding and unbinding energies). Through systematic examinations of the microscopic molecular evolution of the IMS and comparisons between the healing time and the intrinsic timescales, we find 
that the sticky Rouse time serves as a characteristic timescale for the self-adhesion of supramolecular networks, featuring the full recovery of macroscopic interfacial strength. Although we focused on one type of supramolecular network with stickers at the terminals of polymer chains, the conclusion in our work is consistent with the experimental and computational results for other polymeric systems in the literature. For instance, Yan et al. ${ }^{69}$ studied the self-healing of the unentangled supramolecular network formed by the telechelic polyisobutylene with barbituric acid groups at both chains' ends. They found that the terminal Rouse relaxation process is responsible for the self-healing process and $\tau_{\mathrm{eq}} \simeq \tau_{\mathrm{R}}^{\mathrm{s}}$. Considering the role of entanglements in the self-healing of fractured supramolecular networks, the feature of sticky Rouse time means that their recovery of interfacial strength does not rely on the sticky chains completely disengaged from their tubes. This observation is supported by the experiments for nonsticky polymer systems from different research groups in refs ${ }^{70-72}$ Their experimental results showed that the mechanical strength of the interface between two polymer films can be fully recovered long before the disentanglement time. Finally, we need to mention that our results on entanglements recovery at the interface is consistent with the computational observations on the welding between surfaces of highly entangled polymer films in refs. ${ }^{26,28}$ In their MD simulations, the healing time to fully restore the maximum interfacial strength is around 1-3 million $\tau$ when the entanglement areal density at the fracture interface is recovered to around $80 \%$ against the bulk value. ${ }^{26,27}$ Although they did not mention in their works, due to the different focus on the problem, the timescale of 1-3 million $\tau$ is just comparable with the Rouse time $\tau_{\mathrm{R}}$ for their computational system. ${ }^{28}$ Therefore, by pointing out the sticky Rouse time as a characteristic timescale for the self-adhesion process, our conclusion might also be applied on the self-healing/adhesion of other systems, which restore their interfacial strength through the r-bond and entanglements. We expect more works along this direction to test this hypothesis.

Conclusions. In summary, our simulation results provide a clear microscopic picture of the evolution of IMSs and reveal their relations with the macroscopic interfacial strength of supramolecular polymer networks during self-adhesion. More importantly, we further consider the healing time in the context of intrinsic relaxation timescales of supramolecular networks. The sticky Rouse time is found to feature the selfadhesion process of all fractured systems, denoting the full recovery of interfacial strength. These findings will help to establish a molecular theory for the self-adhesion mechanics of supramolecular polymer networks and guide their molecular design. $^{73-76}$

\section{ASSOCIATED CONTENT}

\section{SI Supporting Information}

The Supporting Information is available free of charge at https://pubs.acs.org/doi/10.1021/acs.macromol.1c00335.

Properties of the bulk systems; self-adhesion process of unentangled and entangled supramolecular networks; and segment survival probability functions for entangled supramolecular networks (PDF)

\section{AUTHOR INFORMATION}

\section{Corresponding Author}

Ying Li - Department of Mechanical Engineering and Polymer Program, Institute of Materials Science, University of Connecticut, Storrs 06269, Connecticut, United States; 다. orcid.org/0000-0002-1487-3350; Email: yingli@ engr.uconn.edu

\section{Authors}

Zhiqiang Shen - Department of Mechanical Engineering, University of Connecticut, Storrs 06269, Connecticut, United States; orcid.org/0000-0003-0804-2478

Huilin Ye - Department of Mechanical Engineering, University of Connecticut, Storrs 06269, Connecticut, United States; (1) orcid.org/0000-0002-8041-4056

Qiming Wang - Sonny Astani Department of Civil and Environmental Engineering, University of Southern California, Los Angeles, Los Angeles, California 90089, United States

Martin Kröger - Department of Materials, Polymer Physics, ETH Zürich, Zurich CH-8093, Switzerland; ㅇo이.org/ 0000-0003-1402-6714

Complete contact information is available at:

https://pubs.acs.org/10.1021/acs.macromol.1c00335

\section{Notes}

The authors declare no competing financial interest.

\section{ACKNOWLEDGMENTS}

This work was mainly supported by the National Science Foundation CMMI-1762661 (to Y.L.) and CMMI-1762567 (to Q.W.). Y.L. also would like to thank the support from the Interdisciplinary Multi-Investigator Materials Proposals (IMMP) program of the Institute of Materials Science at the University of Connecticut. Q.W. also acknowledges the funding support from the National Science Foundation (CMMI-1943598) and the Air Force Office of Scientific Research (FA9550-18-1-0192). The contribution by M.K. was promoted by the Swiss National Science Foundation through grant 200021L-185052 and by the Swiss National Supercomputing Centre through grant s987. The authors acknowledge the Texas Advanced Computing Center (TACC) at The University of Texas at Austin for providing HPC resources (Frontera project and the National Science Foundation Award 1818253) that have contributed to the research results reported within this paper. This work was partially supported by a fellowship grant (to Z.S. and H.Y.) from GE's Industrial Solutions Business Unit under a GE-UConn partnership agreement. The views and conclusions contained in this document are those of the authors and should not be interpreted as necessarily representing the official policies, either expressed or implied, of Industrial Solutions or UConn. This research benefited in part from the computational resources and staff contributions provided by the Booth Engineering Center for Advanced Technology (BECAT) at the University of Connecticut.

\section{REFERENCES}

(1) Cordier, P.; Tournilhac, F.; Soulié-Ziakovic, C.; Leibler, L. Selfhealing and thermoreversible rubber from supramolecular assembly. Nature 2008, 451, 977-980.

(2) Holten-Andersen, N.; Harrington, M. J.; Birkedal, H.; Lee, B. P.; Messersmith, P. B.; Lee, K. Y. C.; Waite, J. H. pH-induced metal- 
ligand cross-links inspired by mussel yield self-healing polymer networks with near-covalent elastic moduli. Proc. Natl. Acad. Sci. U.S.A. 2011, 108, 2651-2655.

(3) Wei, Z.; Yang, J. H.; Zhou, J.; Xu, F.; Zrínyi, M.; Dussault, P. H.; Osada, Y.; Chen, Y. M. Self-healing gels based on constitutional dynamic chemistry and their potential applications. Chem. Soc. Rev. 2014, 43, 8114-8131.

(4) Taylor, D. L.; in het Panhuis, M. Self-Healing Hydrogels. Adv. Mater. 2016, 28, 9060-9093.

(5) Li, J.; Geng, L.; Wang, G.; Chu, H.; Wei, H. Self-healable gels for use in wearable devices. Chem. Mater. 2017, 29, 8932-8952.

(6) Wojtecki, R. J.; Meador, M. A.; Rowan, S. J. Using the dynamic bond to access macroscopically responsive structurally dynamic polymers. Nat. Mater. 2011, 10, 14-27.

(7) Skene, W. G.; Lehn, J.-M. P. Dynamers: polyacylhydrazone reversible covalent polymers, component exchange, and constitutional diversity. Proc. Natl. Acad. Sci. U.S.A. 2004, 101, 8270-8275.

(8) Li, C.-H.; Wang, C.; Keplinger, C.; Zuo, J.-L.; Jin, L.; Sun, Y.; Zheng, P.; Cao, Y.; Lissel, F.; Linder, C.; et al. A highly stretchable autonomous self-healing elastomer. Nat. Chem. 2016, 8, 618.

(9) Gulyuz, U.; Okay, O. Self-Healing Poly(acrylic acid) Hydrogels with Shape Memory Behavior of High Mechanical Strength. Macromolecules 2014, 47, 6889-6899.

(10) Sun, T. L.; Kurokawa, T.; Kuroda, S.; Ihsan, A. B.; Akasaki, T.; Sato, K.; Haque, M. A.; Nakajima, T.; Gong, J. P. Physical hydrogels composed of polyampholytes demonstrate high toughness and viscoelasticity. Nat. Mater. 2013, 12, 932-937.

(11) Benight, S. J.; Wang, C.; Tok, J. B. H.; Bao, Z. Stretchable and self-healing polymers and devices for electronic skin. Prog. Polym. Sci. 2013, 38, 1961-1977.

(12) Cheng, X.; Pan, J.; Zhao, Y.; Liao, M.; Peng, H. Gel polymer electrolytes for electrochemical energy storage. Adv. Energy Mater. 2018, 8, 1702184.

(13) Brochu, A. B. W.; Craig, S. L.; Reichert, W. M. Self-healing biomaterials. J. Biomed. Mater. Res., Part A 2011, 96, 492-506.

(14) Terryn, S.; Brancart, J.; Lefeber, D.; Van Assche, G.; Vanderborght, B. Self-healing soft pneumatic robots. Sci. Robot. 2017, 2, No. eaan4268.

(15) Yu, K.; Du, H.; Xin, A.; Lee, K. H.; Feng, Z.; Masri, S. F.; Chen, Y.; Huang, G.; Wang, Q. Healable, memorizable, and transformable lattice structures made of stiff polymers. NPG Asia Mater. 2020, 12, 26.

(16) Pena-Francesch, A.; Jung, H.; Demirel, M. C.; Sitti, M. Biosynthetic self-healing materials for soft machines. Nat. Mater. 2020, 19, 1230-1235.

(17) Binder, W. H. Self-healing Polymers: From Principles to Applications; John Wiley \& Sons, 2013.

(18) Stukalin, E. B.; Cai, L.-H.; Kumar, N. A.; Leibler, L.; Rubinstein, M. Self-healing of unentangled polymer networks with reversible bonds. Macromolecules 2013, 46, 7525-7541.

(19) Bose, R. K.; Hohlbein, N.; Garcia, S. J.; Schmidt, A. M.; van der Zwaag, S. Connecting supramolecular bond lifetime and network mobility for scratch healing in poly(butyl acrylate) ionomers containing sodium, zinc and cobalt. Phys. Chem. Chem. Phys. 2015, $17,1697-1704$.

(20) Hornat, C. C.; Urban, M. W. Entropy and interfacial energy driven self-healable polymers. Nat. Commun. 2020, 11, 1028.

(21) Wool, R. P.; O'Connor, K. M. A theory crack healing in polymers. J. Appl. Phys. 1981, 52, 5953-5963.

(22) de Gennes, P. G. Reptation of a polymer chain in the presence of fixed obstacles. J. Chem. Phys. 1971, 55, 572-579.

(23) Doi, M.; Edwards, S. F. The Theory of Polymer Dynamics; Oxford University Press, 1988; Vol. 73.

(24) Wu, D. Y.; Meure, S.; Solomon, D. Self-healing polymeric materials: a review of recent developments. Prog. Polym. Sci. 2008, 33, $479-522$.

(25) Zhang, M. Q.; Rong, M. Z. Theoretical consideration and modeling of self-healing polymers. J. Polym. Sci., Part B: Polym. Phys. 2012, 50, 229-241.
(26) Ge, T.; Pierce, F.; Perahia, D.; Grest, G. S.; Robbins, M. O. Molecular dynamics simulations of polymer welding: Strength from interfacial entanglements. Phys. Rev. Lett. 2013, 110, 098301.

(27) Ge, T.; Robbins, M. O.; Perahia, D.; Grest, G. S. Healing of polymer interfaces: Interfacial dynamics, entanglements, and strength. Phys. Rev. E: Stat., Nonlinear, Soft Matter Phys. 2014, 90, 012602.

(28) Pierce, F.; Perahia, D.; Grest, G. S. Dynamics of polymers across an interface. Europhys. Lett. 2011, 95, 46001.

(29) Jangizehi, A.; Ghaffarian, S. R.; Schmolke, W.; Seiffert, S. Dominance of chain entanglement over transient sticking on chain dynamics in hydrogen-bonded supramolecular polymer networks in the melt. Macromolecules 2018, 51, 2859-2871.

(30) Shabbir, A.; Javakhishvili, I.; Cerveny, S.; Hvilsted, S.; Skov, A. L.; Hassager, O.; Alvarez, N. J. Linear viscoelastic and dielectric relaxation response of unentangled UPy-based supramolecular networks. Macromolecules 2016, 49, 3899-3910.

(31) Tang, S.; Wang, M.; Olsen, B. D. Anomalous self-diffusion and sticky Rouse dynamics in associative protein hydrogels. J. Am. Chem. Soc. 2015, 137, 3946-3957.

(32) Tang, S.; Olsen, B. D. Relaxation Processes in Supramolecular Metallogels Based on Histidine-Nickel Coordination Bonds. Macromolecules 2016, 49, 9163-9175.

(33) Yang, Y.; Urban, M. W. Self-healing polymeric materials. Chem. Soc. Rev. 2013, 42, 7446-7467.

(34) Yamaguchi, M.; Ono, S.; Terano, M. Self-repairing property of polymer network with dangling chains. Mater. Lett. 2007, 61, 13961399.

(35) Mikos, A. G.; Peppas, N. A. Polymer chain entanglements and brittle fracture. J. Chem. Phys. 1988, 88, 1337-1342.

(36) Silvestri, L.; Brown, H. R.; Carrà, S.; Carrà, S. Chain entanglements and fracture energy in interfaces between immiscible polymers. J. Chem. Phys. 2003, 119, 8140-8149.

(37) Davydovich, D.; Urban, M. W. Water accelerated self-healing of hydrophobic copolymers. Nat. Commun. 2020, 11, 5743.

(38) Sing, C. E.; Alexander-Katz, A. Equilibrium structure and dynamics of self-associating single polymers. Macromolecules 2011, 44, 6962-6971.

(39) Parada, G. A.; Zhao, X. Ideal reversible polymer networks. Soft Matter 2018, 14, 5186-5196.

(40) Rubinstein, M.; Semenov, A. N. Dynamics of entangled solutions of associating polymers. Macromolecules 2001, 34, 10581068.

(41) Rubinstein, M.; Semenov, A. N. Thermoreversible gelation in solutions of associating polymers. 2. Linear dynamics. Macromolecules 1998, 31, 1386-1397.

(42) Kremer, K.; Grest, G. S. Dynamics of entangled linear polymer melts: A molecular-dynamics simulation. J. Chem. Phys. 1990, 92, 5057-5086.

(43) Grest, G. S. Communication: Polymer entanglement dynamics: Role of attractive interactions. J. Chem. Phys. 2016, 145, 141101.

(44) Bell, G. Models for the specific adhesion of cells to cells. Science 1978, 200, 618-627.

(45) Xu, G.; Huang, Z.; Chen, P.; Cui, T.; Zhang, X.; Miao, B.; Yan, L.-T. Optimal Reactivity and Improved Self-Healing Capability of Structurally Dynamic Polymers Grafted on Janus Nanoparticles Governed by Chain Stiffness and Spatial Organization. Small 2017, 13, 1603155.

(46) Xu, Z.; Dai, X.; Bu, X.; Yang, Y.; Zhang, X.; Man, X.; Zhang, X.; Doi, M.; Yan, L.-T. Enhanced Heterogeneous Diffusion of Nanoparticles in Semiflexible Networks. ACS Nano 2021, 3, 4608-4616.

(47) Kröger, M. Shortest multiple disconnected path for the analysis of entanglements in two- and three-dimensional polymeric systems. Comput. Phys. Commun. 2005, 168, 209-232.

(48) Kröger, M.; Hess, S. Rheological evidence for a dynamical crossover in polymer melts via nonequilibrium molecular dynamics. Phys. Rev. Lett. 2000, 85, 1128.

(49) Hoy, R. S.; Foteinopoulou, K.; Kröger, M. Topological analysis of polymeric melts: Chain-length effects and fast-converging 
estimators for entanglement length. Phys. Rev. E: Stat., Nonlinear, Soft Matter Phys. 2009, 80, 031803.

(50) Li, Y.; Kröger, M.; Liu, W. K. Nanoparticle effect on the dynamics of polymer chains and their entanglement network. Phys. Rev. Lett. 2012, 109, 118001.

(51) Li, Y.; Tang, S.; Kröger, M.; Liu, W. K. Molecular simulation guided constitutive modeling on finite strain viscoelasticity of elastomers. J. Mech. Phys. Solids 2016, 88, 204-226.

(52) Plimpton, S. Fast parallel algorithms for short-range molecular dynamics. J. Comp. Physiol. 1995, 117, 1-19.

(53) Humphrey, W.; Dalke, A.; Schulten, K. VMD: visual molecular dynamics. J. Mol. Graphics 1996, 14, 33-38.

(54) Kröger, M. Efficient hybrid algorithm for the dynamic creation of wormlike chains in solutions, brushes, melts and glasses. Comput. Phys. Commun. 1999, 118, 278-298.

(55) Sides, S. W.; Grest, G. S.; Stevens, M. J.; Plimpton, S. J. Effect of end-tethered polymers on surface adhesion of glassy polymers. $J$. Polym. Sci., Part B: Polym. Phys. 2004, 42, 199-208.

(56) Irving, J. H.; Kirkwood, J. G. The statistical mechanical theory of transport processes. IV. The equations of hydrodynamics. J. Chem. Phys. 1950, 18, 817-829.

(57) Cromwell, O. R.; Chung, J.; Guan, Z. Malleable and self-healing covalent polymer networks through tunable dynamic boronic ester bonds. J. Am. Chem. Soc. 2015, 137, 6492-6495.

(58) Yount, W. C.; Loveless, D. M.; Craig, S. L. Small-molecule dynamics and mechanisms underlying the macroscopic mechanical properties of coordinatively cross-linked polymer networks. J. Am. Chem. Soc. 2005, 127, 14488-14496.

(59) Mateyisi, M. J.; Sommer, J.-U.; Müller-Nedebock, K. K.; Heinrich, G. Influence of weak reversible cross-linkers on entangled polymer melt dynamics. J. Chem. Phys. 2018, 148, 244901.

(60) Yount, W. C.; Loveless, D. M.; Craig, S. L. Strong means slow: Dynamic contributions to the bulk mechanical properties of supramolecular networks. Angew. Chem., Int. Ed. 2005, 44, 27462748.

(61) McKinnon, D. D.; Domaille, D. W.; Cha, J. N.; Anseth, K. S. Bis-aliphatic hydrazone-linked hydrogels form most rapidly at physiological $\mathrm{pH}$ : identifying the origin of hydrogel properties with small molecule kinetic studies. Chem. Mater. 2014, 26, 2382-2387.

(62) Callies, X.; Véchambre, C.; Fonteneau, C.; Pensec, S.; Chenal, J.-M.; Chazeau, L.; Bouteiller, L.; Ducouret, G.; Creton, C. Linear rheology of supramolecular polymers center-functionalized with strong stickers. Macromolecules 2015, 48, 7320-7326.

(63) Callies, X.; Fonteneau, C.; Véchambre, C.; Pensec, S.; Chenal, J.-M.; Chazeau, L.; Bouteiller, L.; Ducouret, G.; Creton, C. Linear rheology of bis-urea functionalized supramolecular poly(butylacrylate)s: Part I - weak stickers. Polymer 2015, 69, 233-240.

(64) Yu, K.; Xin, A.; Wang, Q. Mechanics of self-healing polymer networks crosslinked by dynamic bonds. J. Mech. Phys. Solids 2018, 121, 409-431.

(65) Rubinstein, M.; Panyukov, S. Elasticity of polymer networks. Macromolecules 2002, 35, 6670-6686.

(66) Davidson, J. D.; Goulbourne, N. C. A nonaffine network model for elastomers undergoing finite deformations. J. Mech. Phys. Solids 2013, 61, 1784-1797.

(67) When $\tau_{\text {relax }} \ll \tau_{\mathrm{b}}$, the $\tau_{\mathrm{R}}^{\mathrm{s}}$ is determined by $\tau_{\mathrm{b}}$, ${ }^{40}$ i.e., $\propto \tau_{\mathrm{R}}^{\mathrm{s}} \mathrm{N} 0$. When $\tau_{\text {relax }}$ is comparable to $\tau_{\mathrm{b}}$, the $\tau_{\mathrm{R}}^{\mathrm{s}}$ is influenced by both relaxation of precursor polymers and reversible bonds. According to ref 59., $\propto$ $N^{2} \tau_{\mathrm{R}}^{s}(1+\xi \mathrm{L} / \mathrm{N})$, where $\xi \mathrm{L} \propto \tau_{\mathrm{b}}$ is the additional friction induced by reversible bonds. Based on this equation, we should have $\propto \mathrm{N}^{2} \tau_{\mathrm{R}}^{\mathrm{s}}$, if $\tau_{\text {relax }} \gg \tau_{\mathrm{b}}$ for extremely long chains.

(68) Stephanou, P. S.; Baig, C.; Tsolou, G.; Mavrantzas, V. G.; Kröger, M. Quantifying chain reptation in entangled polymer melts: Topological and dynamical mapping of atomistic simulation results onto the tube model. J. Chem. Phys. 2010, 132, 124904.

(69) Yan, T.; Schröter, K.; Herbst, F.; Binder, W. H.; ThurnAlbrecht, T. Unveiling the molecular mechanism of self-healing in a telechelic, supramolecular polymer network. Sci. Rep. 2016, 6, 32356.
(70) Wool, R. P. Polymer Interfaces: Structure and Strength; Hanser Publishers, 1995.

(71) Schnell, R.; Stamm, M.; Creton, C. Mechanical properties of homopolymer interfaces: Transition from simple pullout to crazing with increasing interfacial width. Macromolecules 1999, 32, 34203425.

(72) McGraw, J. D.; Fowler, P. D.; Ferrari, M. L.; Dalnoki-Veress, K. Relaxation of non-equilibrium entanglement networks in thin polymer films. Eur. Phys. J. E 2013, 36, 7.

(73) Zhao, X. Multi-scale multi-mechanism design of tough hydrogels: building dissipation into stretchy networks. Soft Matter 2014, 10, 672-687.

(74) Lin, S.; Zhao, X. Fracture of polymer networks with diverse topological defects. Phys. Rev. E: Stat., Nonlinear, Soft Matter Phys. 2020, 102, 052503.

(75) Hui, C.-Y.; Long, R. A constitutive model for the large deformation of a self-healing gel. Soft Matter 2012, 8, 8209-8216.

(76) Guo, J.; Liu, M.; Zehnder, A. T.; Zhao, J.; Narita, T.; Creton, C.; Hui, C.-Y. Fracture mechanics of a self-healing hydrogel with covalent and physical crosslinks: A numerical study. J. Mech. Phys. Solids 2018, 120, 79-95.

(77) Cui, Z.; Brinson, L. C. Thermomechanical properties and deformation of coarse-grained models of hard-soft block copolymers. Physical Review E 2013, 88 (2), 022602. 